\title{
BRGÖ 2016
}

Beiträge zur Rechtsgeschichte Österreichs

\section{Vorwort}

"Seine kaiserliche königliche apostolische majestät haben in fortsetzung des justiz-regulirungssistems dem magistrat der stadt Wien als das allgemeine hierortige forum non nobilium mit erstem november dies jahrs anzufangen [...] zu sistemisiren geruhet. [...] In betref des senats in ziviljustizgeschäften aber gebühret dem magistrat die gerichtsbarkeit in streitsachen sowohl als in den geschäften des edlen richteramts in seinem ganzen umfange über alle inner den grenzen der linien der kaiserlichen residenzstadt Wien befindliche unadeliche partheyen, ohne unterschied ihres begleitenden karakters, dienstes oder sonstigen geistlichen und weltlichen würden, soweit dieselbe nicht a. der gerichtsbarkeit des reichshofraths und der reichskanzley nach vorschrift des patents vom $10^{\text {ten }}$ julius $1770, b$. der gerichtsbarkeit der militärgerichtsbehörde nach maaß der bestehenden militärverfaßung, $c$. der gerichtsbarkeit einer berggerichtlichen instanz nach vorschrift des patents vom $1^{\text {ten }}$ november 1781, d. der gerichtsbarkeit des merkantil- und wechselgerichts nach anordnung des patents vom $6^{\text {ten }}$ december 1781, oder e. einer inner den linien befindlichen grundherrschaft und ständischen dominio gemäß der landesverfaßung unterstehen, wie auch $f$. mit auschluß der unterthanen der Ottomannischen pforte. "1

Mit dem hier auszugsweise wiedergegebenen Patent Josephs II. von 1783 erfolgte eine der bedeutendsten Reformen des Gerichtswesens für Wien, deren Auswirkungen bis heute spürbar sind: Beseitigte es doch die bisherige, von der tradierten Ständeordnung geprägte Gerichtsorganisation und schuf ein einheitliches System, das die Grundlage für die heutige ordentliche Gerichtsbarkeit darstellte. Deren Entwicklung vom vorhin genannten "Senat in Ziviljustizgeschäften" hin zu den heute in Wien tätigen Zivilgerichten wird im gegenständlichen Tagungsband von Alfred Waldstätten dargestellt, die Geschichte des Landesgerichts für Strafsachen Wien, das seinen Vorläufer im josephinischen "Senat in Kriminaljustizgeschäften" hat, von Friedrich Forsthuber. Dagegen wurde 1783 zum Beispiel die autonome Universitätsgerichtsbarkeit beseitigt und die Gerichtsbarkeit der katholischen Konsistorien massiv beschränkt. Ein letzter Rest der einstigen Universitätsgerichtsbarkeit, die Disziplinargerichtsbarkeit der Universität Wien wird von Kamila Staudigl-Ciechowicz behandelt; Andrea Griesebner stellt Ergebnisse eines Forschungsprojekts zu den Konsistorien vor.

\footnotetext{
${ }^{1}$ Wiener Magistratsverfassung v. 16. 8. 1783, in: Peter CsENDES (Hg.), Die Rechtsquellen der Stadt Wien (= Fontes Iuris 9, Wien-Köln-Graz 1986) Nr. 89.
} 
Der Quellenausschnitt zeigt ebenso, dass auch nach dieser Reform eine Reihe von Sondergerichten bestehen blieben. So insbesondere die von Stefan Wedrac behandelte Handelsgerichtsbarkeit, die von Martin Moll dargestellte Militärgerichtsbarkeit oder das von Christoph Schmetterer untersuchte Sondergericht des Kaiserhauses, das Obersthofmarschallamt.

Sie alle waren Gegenstand der Tagung, die am 11. und 12. Dezember 2015 im Theatersaal der ÖAW stattfand und deren Beiträge in diesem Tagungsband vereint sind. Es galt, die aus heutiger Sicht schier unglaubliche Gerichtsvielfalt, die in Wien einst existierte, zu analysieren, den Weg hin zur modernen Gerichtsorganisation zu erkennen und die Entwicklung des modernen Gerichtsbegriffes zu untersuchen. Dessen Wesen wird heute besonders darin erblickt, dass die Richter unabhängig, unversetzbar und unabsetzbar sind (Art. 87 und Art. 88 Bundes-Verfassungsgesetz). Derartige Kriterien können für die Zeit vor 1848 kaum als Maßstab für den Gerichtsbegriff verwendet werden. Und auch im 20. Jahrhundert waren diese Säulen des Rechtsstaates nicht immer aufrecht; Ilse Reiter-Zatloukal behandelt die (Un)Abhängigkeit der Richter in der Zeit der Diktaturen 1933-1945.

Die Tagung nahm auch auf den Umstand Rücksicht, dass Wien seit Jahrhunderten eine überregionale Bedeutung gerade auch auf dem Gebiet der Gerichtsbarkeit hatte. Dieser Aspekt wurde namentlich in den Beiträgen von Christian Neschwara über die Oberste Justizstelle, von István Fazekas über die Ungarische Hofkanzlei, von Petr Mat’a über die Böhmische Hofkanzlei und von Eva Ortlieb über den Reichshofrat deutlich.

Auch beschränkte und beschränkt sich die "Dritte Gewalt", wie die Justiz oft genannt wird, kaum jemals auf das Zivil- und Strafrecht. Vielmehr existierte eine alte Tradition der Verwaltungsrechtspflege, die von Ulrike Müßig dargestellt wird, während Thomas Olechowski die Entwicklung hin zur modernen Verwaltungsgerichtsbarkeit behandelt.

Schließlich ist "Gerichtsbarkeit" nicht allein ein staatliches Phänomen. Vielmehr erfreuen sich gerade in jüngster Zeit verschiedene Schiedsgerichtsbarkeiten großer Beliebtheit, auch haben bestimmte Stände eigene (Disziplinar)Gerichtsbarkeiten entwickelt; von ihnen wird jene der Wirtschaftstreuhänder von Erich Kodek, jene der Ärzte von Michael Memmer behandelt.

Die Darstellung der Gerichtsbarkeiten muss notwendig lückenhaft bleiben; eine enzyklopädische Darstellung aller Gerichte und gerichtsähnlicher Behörden, die jemals in Wien tätig waren, wurde aber auch nicht beabsichtigt. (Schon die Erfassung nur der ordentlichen Gerichte von der Mitte des 18. Jahrhunderts bis in die Gegenwart resultierte in einem mehrere hundert Seiten umfassenden Handbuch. ${ }^{2}$ ) Die Tagung konnte aber wesentliche Schlaglichter auf dieses so große Themengebiet werfen und wird hoffentlich zu weiteren Forschungen dazu

${ }^{2}$ Alfred WALDSTÄTtEN, Staatliche Gerichte in Wien seit Maria Theresia. Beiträge zu ihrer Geschichte. Ein Handbuch (Innsbruck-Wien 2011). 
anregen. Nicht nur einzelne Gerichte wären es wert, genauer erforscht zu werden, auch das Zusammenwirken der vielen Gerichte und gerichtsähnlichen Institutionen bietet vor allem außerhalb der staatlichen Straf- und Zivilgerichtsbarkeit noch viele Ansätze für rechtshistorische Untersuchungen.

Die Herausgeber danken der Kulturabteilung der Stadt Wien für die großzügige finanzielle Unterstützung sowohl der Tagung als auch des Tagungsbandes. Sie danken Carmen Kleinszig, Andreas Lechner und Harald Trobollowitsch für die Unterstützung bei der Vorbereitung und Durchführung der Tagung, Carina Koplenig, Ramon Pils und Laura Rathmanner für die Hilfe bei der Redaktion und das Lektorat des vorliegenden Bandes. Dank gilt auch Herbert Kalb und Christian Neschwara für den Vorsitz in zwei Panels der Konferenz. Schließlich sei der Präsidentin der phil.-hist. Klasse der Akademie der Wissenschaften, Brigitte Mazohl, und dem Präsidenten des Obersten Gerichtshofs, Eckart Ratz, ganz besonders für die Eröffnung der Tagung gedankt. Dass Eckart Ratz darüber hinaus den Vorsitz in einem Panel übernahm, war ein erfreuliches Zeichen für das Interesse eines hochrangigen Praktikers an der Rechtsgeschichte.

Wien, im Oktober 2016

Thomas OLECHOWSKI

Eva ORTLIEB

Christoph SCHMETTERER 
